\title{
Prospective assessment of catheter- associated bacteriuria clinical presentation, epidemiology, and colonization dynamics in nursing home residents
}

\author{
Chelsie E. Armbruster, ${ }^{1}$ Aimee L. Brauer, ${ }^{1}$ Monica S. Humby, ${ }^{1}$ Jiahui Shao, ${ }^{2}$ and Saptarshi Chakraborty ${ }^{2}$ \\ 'Department of Microbiology and Immunology, Jacobs School of Medicine and Biomedical Sciences, and \\ ${ }^{2}$ Department of Biostatistics, School of Public Health and Health Professions, State University of New York at Buffalo, \\ Buffalo, New York, USA.
}

BACKGROUND. Catheterization facilitates continuous bacteriuria, for which the clinical significance remains unclear. This study aimed to determine the clinical presentation, epidemiology, and dynamics of bacteriuria in a cohort of long-term catheterized nursing home residents.

METHODS. Prospective urine culture, urinalysis, chart review, and assessment of signs and symptoms of infection were performed weekly for 19 study participants over 7 months. All bacteria $\geq 1 \times 10^{3} \mathrm{cfu} / \mathrm{mL}$ were cultured, isolated, identified, and tested for susceptibility to select antimicrobials.

RESULTS. In total, 226 of the 234 urine samples were polymicrobial (97\%), with an average of 4.7 isolates per weekly specimen. A total of 228 urine samples $(97 \%)$ exhibited $\geq 1 \times 10^{6} \mathrm{CFU} /$ $\mathrm{mL}, 220$ (94\%) exhibited abnormal urinalysis, 126 (54\%) were associated with at least 1 possible sign or symptom of infection, and $82(35 \%)$ would potentially meet a standardized definition of catheter-associated urinary tract infection (CAUTI), but only 3 had a caregiver diagnosis of CAUTI. Bacterial isolates (286; 30\%) were resistant to a tested antimicrobial agent, and bacteriuria composition was remarkably stable despite a combined total of 54 catheter changes and 23 weeks of antimicrobial use.

CONCLUSION. Bacteriuria composition was largely polymicrobial, including persistent colonization by organisms previously considered to be urine culture contaminants. Neither antimicrobial use nor catheter changes sterilized the urine, at most resulting in transient reductions in bacterial burden followed by new acquisition of resistant isolates. Thus, this patient population exhibits a high prevalence of bacteriuria coupled with potential indicators of infection, necessitating further exploration to identify sensitive markers of true infection.

FUNDING. This work was supported by the NIH (R00 DK105205, R01 DK123158, UL1 TR001412).

Conflict of interest: The authors have declared that no conflict of interest exists.

Copyright: ( 2021 , Armbruster et al. This is an open access article published under the terms of the Creative Commons Attribution 4.0 International License.

Submitted: September 29, 2020 Accepted: September 1, 2021 Published: September 2, 2021

Reference information: /CI Insight. 2021;6(19):e144775.

https://doi.org/10.1172/jici. insight.144775.

\section{Introduction}

Urinary catheter placement in healthcare settings is a common medical procedure utilized in the treatment of $60 \%$ of critically ill patients, $20 \%$ of those in medical and surgical intensive care units, and $5 \%-22 \%$ of residents in long-term care facilities (1-6). This is particularly true in nursing homes, where $12 \%-15 \%$ of newly admitted individuals have an indwelling urinary catheter and $5 \%-10 \%$ will have long-term urinary catheter use for indications such as chronic pressure ulcers or wounds, traumatic pelvic injury, neurogenic bladder, and low overall functional status $(4,7-9)$. However, long-term urinary catheter use increases the risk of developing symptomatic catheter-associated urinary tract infection (CAUTI) and provides a reservoir for antimicrobial resistant bacteria $(8,10,11)$.

Urinary catheters facilitate the presence of bacteria in urine (bacteriuria) as they bypass many of the natural defenses of the urinary tract. Bacteria that colonize the periurethral area are typically excluded from the urinary tract by a combination of micturition (the regular passing of urine), the physical barrier 
provided by intact urothelial cells and mucin, and innate immune defenses. However, insertion of a catheter damages the urothelial barrier, disrupts normal micturition, and causes retention of a low volume of urine within the bladder, all of which facilitate bacterial growth (12-14). The immune response elicited by the catheter also results in accumulation of host proteins such as fibrinogen, which prime the catheter surface and facilitate bacterial attachment (15-17). The combination of these factors creates a uniquely permissive environment for numerous bacterial species to colonize and potentially establish infection. Indeed, for each day that a urinary catheter is in place, there is a $3 \%-8 \%$ incidence of bacteriuria, and long-term catheterization ( $>28$ days) typically results in continuous bacteriuria $(1,18)$.

Catheterization and resulting bacteriuria are associated with numerous adverse outcomes, such as functional decline, increased hospital stays, inappropriate or inadequate antimicrobial treatment, and an overall increase in mortality rate compared with noncatheterized individuals $(1,6,18-23)$. However, catheter-associated bacteriuria is frequently asymptomatic and can be challenging to distinguish from CAUTI, especially in long-term care settings. Guidelines for diagnosis of CAUTI include the presence of clinical signs and symptoms, in addition to a positive urine culture (24-26). For instance, the 2010 clinical care guidelines of the Infectious Diseases Society of America (IDSA) define CAUTI as a positive urine culture ( $\geq 1 \times 10^{3} \mathrm{CFU} / \mathrm{mL}$ of $\geq 1$ bacterial species) combined with signs or symptoms compatible with urinary tract infection (UTI; fever, rigors, altered mental status, malaise or lethargy with no other identified cause, flank pain, costovertebral tenderness, acute hematuria, or pelvic discomfort) in the absence of an alternate source of infection $(1,27)$. In the absence of these symptoms, a culture with $\geq 1 \times 10^{5} \mathrm{CFU} / \mathrm{mL}$ of $\geq 1$ bacterial species is considered asymptomatic catheter-associated bacteriuria (1). In contrast, the National Health Safety Network (NHSN) surveillance criteria for long-term care facilities define symptomatic CAUTI as having a positive urine culture (no more than 2 species, of which at least 1 must be a bacterium at $\geq 1$ $\times 10^{5} \mathrm{CFU} / \mathrm{mL}$ of urine) combined with at least 1 of the following: (a) fever (single temperature $>100^{\circ} \mathrm{F}$, repeated temperatures $>99^{\circ} \mathrm{F}$, or an increase of $>2^{\circ} \mathrm{F}$ over baseline); (b) rigors; (c) new-onset hypotension ( $<90$ systolic or $<60$ diastolic blood pressure) without an alternate noninfectious cause; (d) new-onset decline in mental or functional status combined with leukocytosis $\left(>14,000\right.$ leukocytes $\left./ \mathrm{mm}^{3}\right)$ and without an alternate diagnosis; (e) new or increased suprapubic tenderness; (f) new or increased costovertebral pain or tenderness; (g) acute pain, swelling, or tenderness of the testes, epididymis, or prostate; or (h) purulent discharge from the catheter insertion site $(25,28)$.

Diagnosis of CAUTI is particularly challenging in older adults, especially those with neurogenic bladder, cognitive impairments, or a high degree of functional dependence. In this population, many signs and symptoms of infection (such as fever and leukocytosis) are infrequent or absent, while others may be subtle or nonspecific (29-38). A further confounding issue is the high prevalence of acute mental status change and confusion in this population $(31,36,39)$, coupled with issues regarding reliable assessment of mental status and nonspecific symptoms (40). To reduce inappropriate antimicrobial treatment, development of antimicrobial resistance, and risk of Clostridioides difficile infection, the 2019 guidelines of the IDSA strongly recommend against screening for or treating asymptomatic bacteriuria in older, functionally or cognitively impaired adults, especially those residing in long-term care facilities, as well as individuals with indwelling urinary catheters (27). The guidelines further indicate that mental status change and bacteriuria without local genitourinary symptoms or systemic symptoms should not be considered to indicate symptomatic UTI in older, functionally or cognitively impaired adults (27). However, nonspecific symptoms, such as change in mental status and confusion, are the most common indications for suspected UTI in nursing home residents and antimicrobial prescription for asymptomatic bacteriuria remains common in this population $(31,41)$.

While several studies have reported the epidemiology of bacteriuria in catheterized individuals $(1,2$, $31,42-54)$, few have conducted a longitudinal assessment of colonization dynamics or included prospective assessment of common indicators of CAUTI. These collective studies have also demonstrated that polymicrobial bacteriuria and CAUTI are common during long-term catheterization, yet few report the full etiology of polymicrobial urine cultures. To address these gaps in knowledge, we conducted a prospective longitudinal assessment of bacteriuria in long-term catheterized nursing home residents from 2 facilities in western New York. Our study had 3 primary goals: (a) determine the clinical presentation of catheter-associated bacteriuria and CAUTI in long-term catheterized nursing home residents; (b) determine the epidemiology of catheter-associated bacteriuria and CAUTI, including carriage of antimicrobial resistant bacteria; and (c) determine the impact of disruptions, such as catheter changes and antimicrobial treatment, on colonization dynamics. 


\section{Results}

Description of study population. Target enrollment was 50 nursing home residents with long-term indwelling urinary catheters ( $\geq 1$ year), but the study was ended early due to COVID-19, with 19 participants followed for a maximum of 7 months each. As summarized in Table 1, the majority of study participants were White (79\%), male (79\%), and had suprapubic catheters (68\%). Study participants exhibited a high level of functional dependence for activities of daily living, with an average physical self-maintenance score (PSMS) of 22 on a scale ranging from 6 to 30 (55). The most common comorbidities were neurogenic bladder $(74 \%)$, hemiplegia $(42 \%)$, diabetes $(32 \%)$, renal disease $(32 \%)$, multiple sclerosis $(26 \%)$, and chronic heart failure (26\%).

Study participants were followed for a total of 260 patient weeks (13 average, range 1-30), with 9 of the 19 participants completing greater than 12 weeks of follow-up. Including baseline samples, 234 urine samples were collected during the study (average 12 per participant, range 1-28). Ten participants (53\%) had at least 1 catheter change between weekly follow-up visits, for a combined total of 54 catheter changes (5 average, range 1-12). Notably, 13 catheter changes occurred due to blockage/obstruction, 5 occurred due to accidental removal/dislodging, and the remaining 36 catheter changes were listed as routine care. Four participants (21\%) experienced a combined total of 8 caregiver-diagnosed infections during follow-up, 3 of which were CAUTIs. Three participants (16\%) had received antibiotics within 30 days prior to their baseline visit, and 6 participants (32\%) received antimicrobial treatment during the course of follow-up for a total of 23 patient weeks of antimicrobial use, including 2 participants who received antimicrobial treatment for 3 CAUTIs.

Epidemiology of catheter-associated bacteriuria. In total, 234 urine samples were collected during 260 study visits. Samples could not be obtained at 26 study visits due to the absence of the participant on a particular visit or the participant not wanting to be disturbed that day. All urine samples were subjected to semiquantitative streak plating for isolation of all distinct colony types and biochemical determination to the species level (see Methods), as well as quantitative dilution plating on HardyCHROM UTI agar to determine total colony-forming units (CFU) per milliliter of urine. Of the 234 urine cultures, $233(99 \%)$ exhibited bacterial growth $>1 \times 10^{3} \mathrm{CFU} / \mathrm{mL}$, with an average of $5.12 \times 10^{6} \mathrm{CFU} / \mathrm{mL}$ (range $5.5 \times 10^{4}$

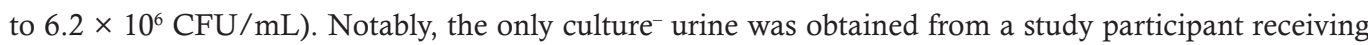
i.v. antimicrobials for CAUTI with bacteremia.

A total of 1092 bacterial isolates were cultured from 233 urine samples, of which $623(57 \%)$ were $\mathrm{Gram}^{-}$and 469 (43\%) were Gram ${ }^{+}$. Select antimicrobial susceptibility was assessed by zone of growth inhibition on Mueller-Hinton agar. All $623 \mathrm{Gram}^{-}$isolates were tested for susceptibility to ciprofloxacin, ceftazidime, ceftazidime with clavulanate, and imipenem; all 168 Staphylococcus aureus isolates were tested for methicillin susceptibility; and all $163 \mathrm{E}$. faecalis isolates were tested for vancomycin susceptibility. Of these combined 954 isolates, $286(30 \%)$ were resistant to at least 1 antimicrobial agent, and resistant organisms were present in urine specimens from 12 of the 19 participants (63\%) (Table 2). The most common resistances were ciprofloxacin (171 of $623 \mathrm{Gram}^{-}$isolates [27\%]), methicillin (81 of $168 \mathrm{~S}$. aureus isolates [48\%]), and ceftazidime (37 of $623 \mathrm{Gram}^{-}$isolates [6\%]). Notably, all isolates that were resistant to ceftazidime were also resistant to ceftazidime with clavulanic acid, indicating production of an extended spectrum $\beta$-lactamase (ESBL). None of the $\mathrm{Gram}^{-}$isolates were resistant to imipenem, and none of the $E$. faecalis isolates were resistant to vancomycin.

Antimicrobial resistance was most prevalent for Proteus mirabilis (69\% of isolates), S. aureus (48\%), Providencia stuartii (44\%), Morganella morganii (37\%), Pseudomonas aeruginosa (29\%), and E. coli (29\%) (Table 2). For most organisms, all sequential isolates from a single participant exhibited the same colony morphology and resistance profile, with the exception of $S$. aureus. Methicillin-resistant $S$. aureus (MRSA) is therefore discussed separately from methicillin-sensitive $S$. aureus (MSSA).

The vast majority of culture ${ }^{+}$urine samples were polymicrobial (226 of $233,97 \%$ ), with an average of 4.7 isolates per weekly urine specimen (range, 1-10). Thirty (13\%) harbored 2 distinct isolates, 24 (11\%) had 3 isolates, 39 (17\%) had 4 isolates, 52 (23\%) had 5 isolates, 41 (18\%) had 6 isolates, $18(8 \%)$ had 7 isolates, and $22(9 \%)$ had 8 or more distinct isolates. A visualization of the full colonization data for each participant at each weekly visit is provided in Supplemental Figure 1 (supplemental material available online with this article; https://doi.org/10.1172/jci.insight.144775DS1), and longitudinal colonization data from 4 study participants are displayed in Figure 1 to align bacteriuria composition with urinalysis and sign and symptom data. As displayed in Table 3, the most common organisms at baseline 


\section{Table 1. Characteristics of study participants}

\begin{tabular}{|c|c|}
\hline Age, mean (SD; range), years & $65(15 ; 43-91)$ \\
\hline Weight, mean (SD; range), pounds & $182(26 ; 117-220)$ \\
\hline PSMS, mean (SD; range) & $22(4 ; 13-27)$ \\
\hline Duration of catheter use (years), mean (SD;range) ${ }^{A}$ & $6.5(4.7 ; 1-17)$ \\
\hline Duration of follow-up (weeks), mean (SD; range) & $13.0(11.0 ; 1-30)$ \\
\hline Number of catheter changes, mean (SD; range) & $2.6(3.6 ; 0-12)$ \\
\hline \multicolumn{2}{|l|}{ Sex } \\
\hline Male & $15(79)$ \\
\hline Female & $4(21)$ \\
\hline \multicolumn{2}{|l|}{ Race } \\
\hline White & $15(79)$ \\
\hline Black or African American & $4(21)$ \\
\hline \multicolumn{2}{|l|}{ Catheter type } \\
\hline Foley & $6(32)$ \\
\hline Suprapubic & $13(68)$ \\
\hline \multicolumn{2}{|l|}{ Indication for catheter use } \\
\hline Urinary retention or outlet obstruction & $19(100)$ \\
\hline \multicolumn{2}{|l|}{ Facility } \\
\hline A & $8(42)$ \\
\hline $\mathrm{B}$ & $11(58)$ \\
\hline \multicolumn{2}{|l|}{ Underlying conditions } \\
\hline Neurogenic bladder & $14(74)$ \\
\hline Hemiplegia & $8(42)$ \\
\hline Diabetes & $6(32)$ \\
\hline Renal disease & $6(32)$ \\
\hline Multiple sclerosis & $5(26)$ \\
\hline Chronic heart failure & $5(26)$ \\
\hline Dementia & $4(21)$ \\
\hline
\end{tabular}

were Enterococcus faecalis (14 of 19 baseline urine specimens, 74\%), P. stuartii (8 of 19, 42\%), E. coli (7 of 19, 37\%), coagulase ${ }^{-}$Staphylococcus (7 of 19,37\%), and P. mirabilis 6 of 19, 32\%). When examined across all study visits, 18 of 19 participants (95\%) were colonized by $E$. faecalis during at least 1 study visit. Eleven of 19 (58\%) were colonized by $P$. mirabilis, 11 of $19(58 \%)$ by coagulase- Staphylococcus, 9 of $19(47 \%)$ by $P$. stuartii, 9 of $19(47 \%)$ by MRSA, 9 of $19(47 \%)$ by MSSA, 8 of $19(42 \%)$ by E. coli, 8 of $19(42 \%)$ by P. aeruginosa, 5 of 19 (26\%) by M. morganii, and 5 of 19 (26\%) by $K$. pneumoniae. Thus, the highest weekly prevalence was observed for E. faecalis (63\%), P. mirabilis (45\%), P. stuartii (43\%), and S. aureus (33\% for methicillin-sensitive isolates and $31 \%$ for methicillin-resistant isolates). The most stable and persistent colonizers were E. faecalis, $P$. mirabilis, $P$. stuartii, and E. coli, while organisms such as $P$. aeruginosa, Providencia rettgeri, Klebsiella pneumoniae, coagulase ${ }^{-}$Staphylococcus, and Serratia marsescens tended to exhibit transient colonization. Consistent with the high percentage of polymicrobial urine samples, the majority of the study participants exhibited polymicrobial bacteriuria during at least 1 study visit (18 of 19, 95\%): 15 of 19 (79\%) exhibited polymicrobial bacteriuria at all weekly visits, 3 of 19 (16\%) mostly had polymicrobial specimens with 1 or 2 monomicrobial samples, and 1 participant only exhibited monomicrobial urine samples (Figure 2).

Microbe-microbe interactions during polymicrobial colonization can have profound implications for risk of developing severe disease (56-61). To quantify such interactions, we first computed the number of occurrences for each microbial species (i.e., the total number of participants colonized by that species) at each time point across the entire longitudinal data set. We then computed Lin's concordance correlation coefficient (62) between the number of occurrences of each microbial pair over the entire longitudinal data set (Supplemental Table 1). Highly concordant cocolonization partners included $P$. mirabilis with $P$. stuartii (80 of 233 culture $^{+}$urine samples [34\%]; 8 of 19 participants [42\%]), P. mirabilis 
Table 2. Prevalence of antimicrobial-resistant isolates

\begin{tabular}{|c|c|c|c|}
\hline Organism & Resistance & Participant prevalence ${ }^{A}$ & Organism prevalence ${ }^{\mathrm{B}}$ \\
\hline Staphylococcus aureus (13 participants, 168 isolates) & Methicillin & $9(69)$ & $81(48)$ \\
\hline Proteus mirabilis (11 participants, 118 isolates) & Ciprofloxacin & $7(64)$ & $81(69)$ \\
\hline \multirow{2}{*}{ Providencia stuartii (9 participants, 111 isolates) } & Ciprofloxacin & $4(44)$ & $27(24)$ \\
\hline & ESBL & $1(11)$ & $22(20)$ \\
\hline Morganella morganii (5 participants, 64 isolates) & Ciprofloxacin & $2(40)$ & $24(37)$ \\
\hline E. coli (8 participants, 89 isolates) & Ciprofloxacin & $1(12)$ & $26(29)$ \\
\hline Proteus vulgaris (3 participants, 37 isolates) & Ciprofloxacin & $2(67)$ & $3(8)$ \\
\hline Klebsiella oxytoca (3 participants, 16 isolates) & ESBL & $1(33)$ & $2(12)$ \\
\hline Enterobacter aerogenes (2 participants, 8 isolates) & ESBL & $1(50)$ & $1(12)$ \\
\hline
\end{tabular}

${ }^{A}$ Number of study participants colonized by the organism with isolates resistant to the listed antimicrobial. ${ }^{B}$ Number of isolates of the organism that were resistant to the listed antimicrobial. Parenthetical numbers represent percentages.

with M. morganii (52 of 233 [22\%]; 4 of 19 [21\%]), M. morganii with P. stuartii (63 of 233 [27\%]; 5 of 19 [26\%]), P. mirabilis with E. faecalis (93 of 233 [40\%]; 9 of 19 [47\%]), and P. stuartii with E. faecalis (86 of 233 [37\%]; 8 of 19 [42\%]). A total of 205 urine samples from all 19 study participants contained at least 1 of these 4 organisms; $121(59 \%)$ samples from 11 participants contained at least 2 of the 4 concordant organisms, $71(35 \%)$ samples from 7 participants contained at least 3 , and $50(24 \%)$ samples from 4 participants included all 4 organisms. It is also notable that cocolonization by these species occurred in both men and women, and with both catheter types (Foley versus suprapubic).

Clinical presentation of catheter-associated bacteriuria. Potential signs and symptoms of infection were prospectively assessed by a nurse at each study visit and were also collected from participant medical records to determine point prevalence at baseline, weekly prevalence, and incidence (Table 4). Assessment included pain (defined as suprapubic and/or costovertebral pain or tenderness), fever (defined as temperature $>$ $99^{\circ} \mathrm{F}$ ), hypotension (defined as $<90$ systolic or $<60$ diastolic blood pressure), acute mental status change (defined as altered level of consciousness, inattention, or disorganized thinking in 1 of 2 minicognitive assessments conducted 30 minutes apart), nausea, lack of appetite, and fatigue. The sign and symptom assessment tool utilized by the study nurses is provided in Supplemental Figure 2. Overall, 16 of 19 (84\%) participants exhibited potential signs and symptoms of infection at 126 of $260(48 \%)$ study visits. A visualization of the sign and symptom data for each participant at each weekly visit is provided in Supplemental Figure 3, and data aligned to bacteriuria and urinalysis results from 4 participants are displayed in Figure 1.

The most common clinical presentations at baseline were hypotension ( 3 of 19, 16\%), fatigue ( 3 of 19 , $16 \%$ ), and lack of appetite ( 3 of 19, 16\%). Altered mental status, fatigue, and lack of appetite exhibited the highest weekly prevalence and incidence, while fever, chills, and leukocytosis were absent at baseline, had a low weekly prevalence and incidence, and were typically only present at a single study visit. Lin's concordance correlation coefficients between the number of occurrences of sign and symptom pairs across the entire longitudinal data set displayed a high degree of concordance between nausea, lack of appetite, fatigue or tiredness, and pain (Supplemental Table 2). Of the 14 participants who completed at least 4 weeks of follow-up, 3 (21\%) were asymptomatic for the vast majority of visits, $3(21 \%)$ experienced 1 or 2 symptomatic episodes that lasted approximately 3 weeks (including 3 of the 4 individuals who experienced caregiver-diagnosed infections), 4 (29\%) experienced frequent multiweek stretches of potential signs or symptoms of infection interspersed with 1 or 2 asymptomatic weeks, and 4 (29\%) exhibited potential signs or symptoms of infection at every study visit. These data highlight some of the challenges for diagnosing CAUTI in long-term catheterized nursing home residents, including the high prevalence and subjective nature of constitutional signs and symptoms of infection (such as nausea, lack of appetite, and fatigue) in this patient population.

Due to the high burden of bacteriuria and possible signs and symptoms of infection in this population, 82 of $260(31 \%)$ study visits would potentially meet the IDSA CAUTI criteria $(1,27)$. If only new-onset symptoms are considered, this number is reduced to 51 of $260(20 \%)$. Only 6 study visits would meet the 

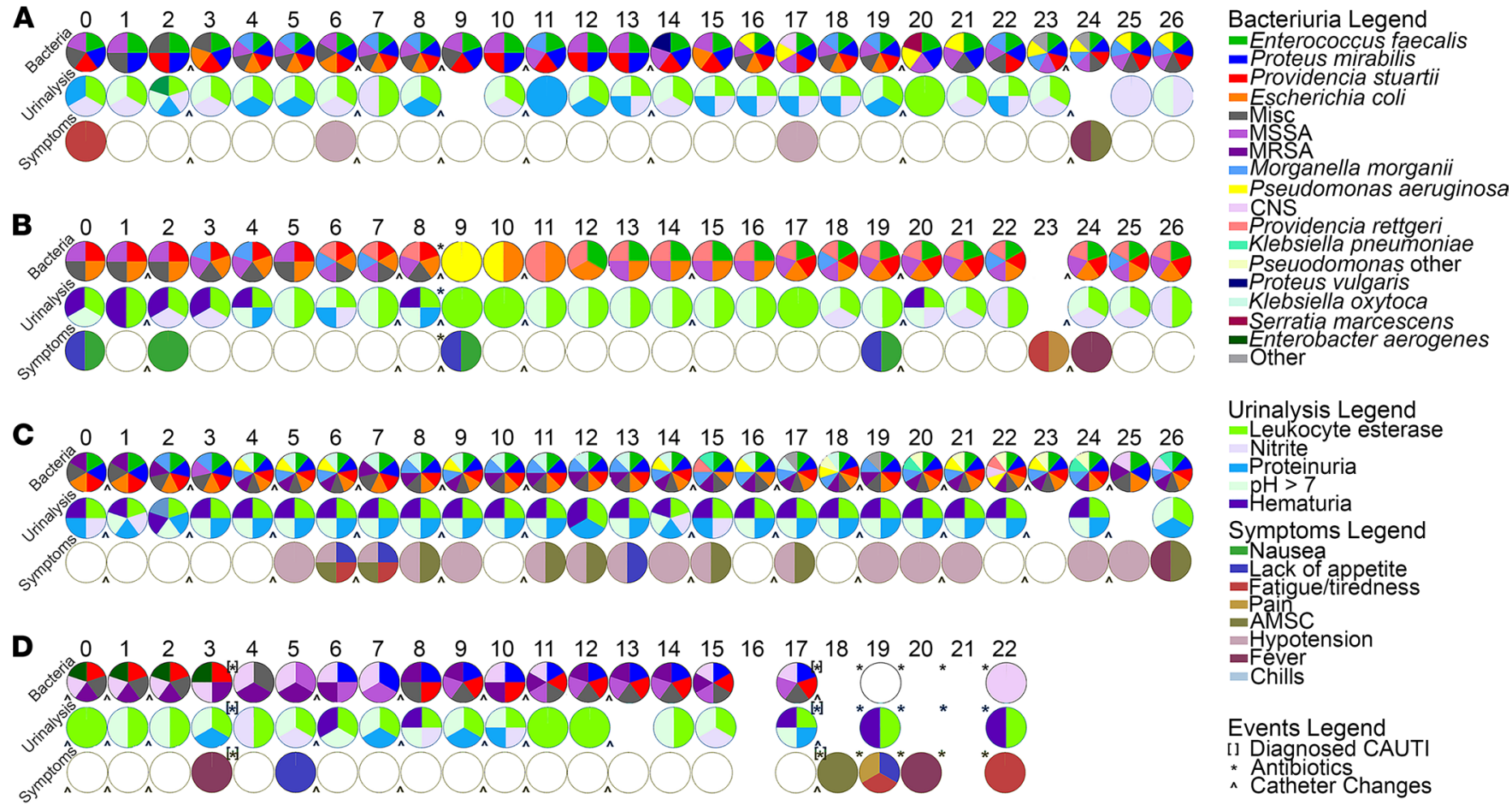

Figure 1. Epidemiology and dynamics of catheter-associated bacteriuria. The complete longitudinal data for 4 study participants (letters) at each study visit (numbers, starting with 0 for baseline) are presented. The first row of pie charts displays the bacterial species that were identified by standard culture methods at each visit, the second row displays the urinalysis test strip results, and the third row displays the potential signs and symptoms of infection that were present at each study visit. White circles with black outlines indicate study visits at which assessments were negative, while empty spaces indicate study visits at which a particular assessment could not be made. Brackets indicate a caregiver-diagnosed CAUTI, asterisks indicate antimicrobial use, and the ^ symbol indicates when the participant's catheter was changed. Misc, Gram+ bacteria that were PYR-Catalase-Streptex; MSSA, methicillin-sensitive Staphylococcus aureus; MRSA, methicillin-resistant Staphylococcus aureus; CNS, coagulase- Staphylococcus; Pseudomonas other, Gram- bacteria with good identification to genus via API-20E but were not P. aeruginosa by PCR; Other, any Gram organism that could not be identified by API-20E.

more strict NHSN surveillance definition for CAUTI $(25,28)$ due to the stringent cutoff of $\leq 2$ organisms in the urine culture and the requirement that acute mental status change must be accompanied by leukocytosis (which was not prospectively assessed and only reported in the medical records of 3 study participants). Importantly, none of the 3 caregiver-diagnosed CAUTIs would meet the NHSN surveillance criteria since all 3 involved $>2$ organisms. However, 2 of the 3 diagnosed CAUTIs involved bacteremia with 1 of the bacterial species present in urine (Supplemental Table 3), indicating that these cases likely represent true CAUTI. Supplemental Table 3 also demonstrates substantial concordance between (a) the bacterial culture results and sign and symptom analysis conducted by the study team and (b) those reported in the participants' medical records. If urine cultures containing 3 or more organisms are permitted for the NHSN criteria, the number of visits that would meet the NHSN CAUTI surveillance definition increases to 27 and includes all 3 caregiver-diagnosed CAUTIs. Taken together, prospective weekly assessment of possible signs and symptoms of CAUTI revealed that numerous potential indicators of infection are common in nursing home residents with long-term catheters and fluctuate in duration, which may further confound the utility of these indicators for distinguishing CAUTI from asymptomatic bacteriuria in this patient population.

All urine samples were also subjected to urinalysis via a 10-parameter urine reagent test strip to determine the point prevalence of parameters that are often considered to be suggestive of UTI, along with weekly prevalence and incidence (Table 5). A visualization of the urinalysis data for each participant at each weekly visit is provided in Supplemental Figure 4, and it is aligned with bacteriuria and symptom data for 4 participants in Figure 1. The most common urinalysis findings at baseline were a positive result for leukocyte esterase (17 of 19 participants, 89\%), nitrites (10 of 19,53\%) hematuria (8 of 19 , $42 \%$ ), and proteinuria (5 of 19, 26\%). The highest weekly prevalence was observed for leukocyte esterase, proteinuria, and nitrites. Leukocyte esterase and high $\mathrm{pH}$ were highly persistent and typically identified in multiple consecutive urine samples, while hematuria and ketones were more likely to be present at a 
Table 3. Epidemiology of catheter-associated bacteriuria

\begin{tabular}{|c|c|c|c|c|c|c|c|c|}
\hline & $\begin{array}{c}\text { Baseline } \\
\text { occurrence }\end{array}$ & $\begin{array}{c}\text { Point } \\
\text { prevalence }^{A}\end{array}$ & Patient weeks & $\begin{array}{c}\text { Weekly } \\
\text { prevalence }^{\text {B }}\end{array}$ & New episodes & Incidence ${ }^{c}$ & $\begin{array}{c}\text { Number } \\
\text { of 1-week } \\
\text { episodes }\end{array}$ & $\begin{array}{c}\text { Proportion } \\
\text { of } 1 \text {-week } \\
\text { episodes }^{D}\end{array}$ \\
\hline Enterococcus faecalis & 14 & 0.74 & 163 & 0.63 & 4 & 0.02 & 0 & 0.00 \\
\hline Proteus mirabilis & 6 & 0.32 & 118 & 0.45 & 5 & 0.02 & 0 & 0.00 \\
\hline Providencia stuartii & 8 & 0.42 & 111 & 0.43 & 6 & 0.02 & 0 & 0.00 \\
\hline E. coli & 7 & 0.37 & 89 & 0.34 & 3 & 0.01 & 0 & 0.00 \\
\hline MSSA & 5 & 0.26 & 87 & 0.33 & 8 & 0.03 & 2 & 0.25 \\
\hline MRSA & 5 & 0.26 & 81 & 0.31 & 12 & 0.05 & 5 & 0.42 \\
\hline Pseudomonas aeruginosa & 4 & 0.21 & 66 & 0.25 & 18 & 0.07 & 6 & 0.33 \\
\hline Morganella morganii & 2 & 0.11 & 64 & 0.25 & 14 & 0.05 & 4 & 0.29 \\
\hline CNS & 7 & 0.37 & 46 & 0.18 & 12 & 0.05 & 6 & 0.50 \\
\hline Providencia rettgeri & 1 & 0.05 & 43 & 0.17 & 9 & 0.03 & 6 & 0.67 \\
\hline Proteus vulgaris & 0 & 0.00 & 37 & 0.14 & 3 & 0.01 & 1 & 0.33 \\
\hline Klebsiella pneumoniae & 2 & 0.11 & 28 & 0.11 & 7 & 0.03 & 3 & 0.43 \\
\hline Klebsiella oxytoca & 0 & 0.00 & 16 & 0.06 & 3 & 0.01 & 0 & 0.00 \\
\hline Serratia marsescens & 1 & 0.05 & 14 & 0.05 & 2 & 0.01 & 2 & 1.00 \\
\hline Enterobacter aerogenes & 2 & 0.11 & 8 & 0.03 & 0 & 0.00 & 0 & NA \\
\hline GBS & 2 & 0.11 & 2 & 0.01 & 0 & 0.00 & 0 & NA \\
\hline
\end{tabular}

MSSA, methicillin-sensitive Staphylococcus aureus; MRSA, methicillin-resistant Staphylococcus aureus; CNS, coagulase- Staphylococcus; GBS, Group B Streptococcus. ${ }^{A}$ Number of baseline urine specimens with organism divided by the total number of baseline urine samples. ${ }^{B}$ Number of patient weeks the organism was detected divided by total number of patient weeks. 'Number of new acquisitions of the organism divided by total number of patient weeks. ${ }^{D}$ Number of episodes lasting only 1 week divided by the number of new episodes (excluding the first and last study visits).

single study visit. Overall, 226 of the 227 urine specimens tested (99\%) had a urinalysis result that could be suggestive of infection in a noncatheterized individual and may therefore influence perception of urine culture results. While this is likely just a reflection of the ubiquitous bacteriuria experienced by this patient population, these data underscore the need for caution when interpreting urinalysis results and possible signs and symptoms of UTI in nursing home residents with long-term indwelling catheters.

Impact of catheter changes and antibiotic use on colonization dynamics and symptom burden. The epidemiology of bacteriuria was remarkably consistent across consecutive study visits, often with only minor or transient perturbations. We therefore sought to determine the impact of catheter changes and antimicrobial use on colonization dynamics for all instances with at least 3 weeks of postevent follow-up (Table 6). Eight participants with at least 12 weeks of follow-up had an average of 5.75 catheter changes each (range $0-12$ ). Catheter changes resulted in a combined loss of 28 isolates, with an average loss of 3.5 per participant (Table 6). However, 20 of the 28 isolates (71\%) were regained within the subsequent 4 weeks, indicating that catheter changes were not effective in reducing bacteriuria in the long-term. Strikingly, 23 new isolates were acquired after catheter changes, for an average of 2.9 new isolates per participant gained from catheter changes. Altogether, catheter changes resulted in a net increase of 1.9 isolates, indicating that changing the catheter was more likely to introduce new organisms than reduce colonization by existing organisms. Furthermore, 4 participants (50\%) acquired at least 1 new isolate resistant to a tested antimicrobial after a catheter change: 5 of the 23 new acquisitions were resistant organisms (22\%), resulting in $28 \%$ average incidence of new acquisition of resistant organisms following catheter changes. Importantly, there was no common source of transmission of new resistant organisms among these 4 participants as 2 resided at one facility and 2 resided at another facility, and all were from different units on different floors within each facility.

In addition to catheter changes, 3 participants with at least 12 weeks of follow-up each received at least 1 patient week of antimicrobial treatment with at least 3 weeks of subsequent follow-up (Table 6). Treatment resulted in a combined loss of 8 isolates, with an average loss of 2.7 colonizing isolates per participant. Similar to catheter changes, 4 of the 8 isolates $(50 \%)$ were regained within the subsequent 3 weeks, and 5 new isolates were acquired (average of 1.7 per participant) resulting in a net increase of 0.3 isolates after antimicrobial treatment. One of the 5 new isolates was resistant to a tested antimicrobial (20\%), resulting in a $17 \%$ average incidence of new acquisition of resistant organisms following treatment. 


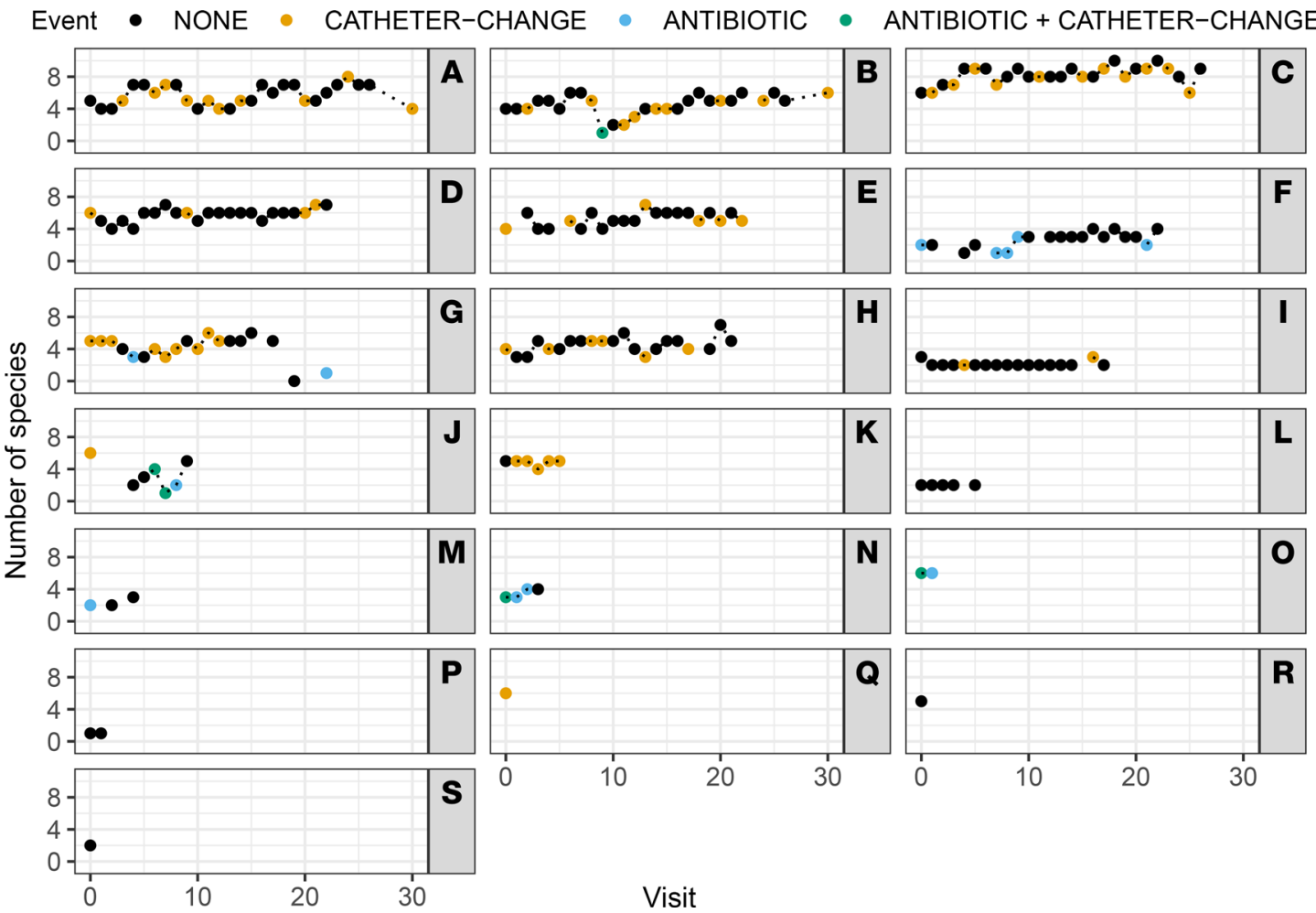

Figure 2. Impact of catheter changes and antibiotic use on colonization density. The total number of unique bacterial species isolated from each weekly urine sample (0-10 species) are displayed for all study participants ( $A-S)$ across each study visit (0-30 visits). Symbol color indicates whether an event occurred since the prior visit that could impact colonization density.

It is also notable that 15 of $27(56 \%)$ study visits, during which the NHSN CAUTI criteria would be met if bacteriuria with $\geq 3$ organisms is permitted, occurred within 10 days after a catheter change or antibiotic use, including all 3 of the caregiver-diagnosed CAUTIs. To further explore the impact of catheter changes and antibiotic use on colonization dynamics and symptom burden, 2 multivariate Bayesian multilevel models were used to analyze the entire data set to determine the contribution of parameters at any given visit to the likelihood of a specific microbe (Supplemental Figure 5) or symptom (Supplemental Figure 6) being present at the following study visit. The Bayesian models coherently address missingness in the data and provide probabilistic quantification of modeling uncertainties (see Methods). For bacterial colonization, the main contributor to colonization by a specific microbe at any given study visit was the presence of that same microbe at the prior study visit, which reflects the overall high degree of stability in colonization. Interestingly, neither antibiotic use nor catheter changes significantly altered the likelihood of colonization by any specific microbe in this analysis, although antibiotic use resulted in a trend toward decreased likelihood of colonization by MSSA, $P$. rettgeri, $P$. stuartii, M. morganii, P. mirabilis, E. faecalis, and miscellaneous PYR-Catalase- $\mathrm{Gram}^{+}$isolates, as well as a trend toward increased likelihood of colonization by $P$. aeruginosa and coagulase- Staphylococcus species. In agreement with our qualitative assessment of the impact of catheter changes and antibiotic treatment on colonization over time (Table 6), neither event significantly reduced the total number of bacterial species present in urine samples from week to week (the 95\% posterior credible intervals for the OR are 0.9994-1.0022 for catheter changes and 0.5546-1.0003 for antibiotic treatment; Figure 2). Similar results were observed for analysis of possible signs and symptoms of infection, with the main contributor to the presence of most symptoms at any given study visit being the presence of that same symptom at the prior study visit, especially for hypotension, pain, fatigue or tiredness, and acute mental status change. Antibiotic use resulted in a slight trend toward increased likelihood of nausea and chills, while catheter changes had no apparent impact in this analysis.

\section{Discussion}

CAUTIs are common in nursing home residents with long-term indwelling catheters and the leading cause of antimicrobial prescriptions in this population (63). However, it is estimated that approximately one-third 
Table 4. Clinical presentation of catheter-associated bacteriuria

\begin{tabular}{|c|c|c|c|c|c|c|c|c|}
\hline & $\begin{array}{c}\text { Baseline } \\
\text { occurrence }\end{array}$ & $\begin{array}{c}\text { Point } \\
\text { prevalence }^{A}\end{array}$ & Patient weeks & $\begin{array}{c}\text { Weekly } \\
\text { prevalence }^{\mathrm{B}}\end{array}$ & New episodes & Incidence ${ }^{c}$ & $\begin{array}{c}\text { Number } \\
\text { of 1-week } \\
\text { episodes }\end{array}$ & $\begin{array}{c}\text { Proportion } \\
\text { of 1-week } \\
\text { episodes }^{D}\end{array}$ \\
\hline$\geq 1 \times 10^{5} \mathrm{CFU} / \mathrm{mL}$ & 19 & 1.00 & 231 & 0.99 & 1 & 0.00 & 0 & 0.00 \\
\hline Hypotension & 3 & 0.16 & 36 & 0.14 & 14 & 0.05 & 7 & 0.50 \\
\hline Fatigue & 3 & 0.16 & 46 & 0.18 & 13 & 0.05 & 8 & 0.62 \\
\hline Altered mental status ${ }^{\mathrm{E}}$ & 2 & 0.11 & 48 & 0.18 & 26 & 0.10 & 14 & 0.54 \\
\hline Lack of appetite & 3 & 0.16 & 43 & 0.17 & 17 & 0.07 & 12 & 0.71 \\
\hline Pain $^{F}$ & 2 & 0.11 & 38 & 0.15 & 10 & 0.04 & 6 & 0.60 \\
\hline Nausea & 2 & 0.11 & 31 & 0.12 & 11 & 0.04 & 5 & 0.45 \\
\hline Fever $^{\complement}$ & 0 & 0.00 & 11 & 0.04 & 11 & 0.04 & 11 & 1.00 \\
\hline Chills & 0 & 0.00 & 10 & 0.04 & 7 & 0.03 & 4 & 0.57 \\
\hline Leukocytosis & 0 & 0.00 & 2 & 0.01 & 2 & 0.01 & 2 & 1.00 \\
\hline
\end{tabular}

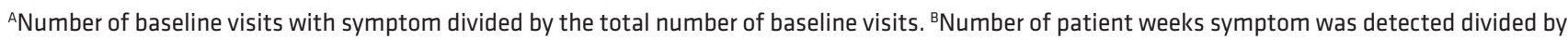
total number of patient weeks. 'Number of new instances of symptom divided by total number of patient weeks. ${ }^{\mathrm{D}} \mathrm{Number}$ of episodes lasting only 1 week divided by the number of new episodes (excluding the first and last study visits). ${ }^{E}$ Altered mental status was assessed by bCAM and DTS (see Methods).

FPain refers to costovertebral or suprapubic pain or tenderness. ${ }^{6}$ Fever was defined as having a temperature $>99^{\circ} \mathrm{F}$.

of CAUTIs are misdiagnosed asymptomatic bacteriuria, for which antimicrobial therapy is not considered to be beneficial $(64,65)$. Part of the discrepancy is due to the challenges of diagnosing CAUTI, especially in a patient population that frequently presents with atypical symptoms. While our sample size was small, our study clearly demonstrates that nursing home residents with long-term catheters routinely have bacteriuria $>1 \times 10^{5} \mathrm{CFU} / \mathrm{mL}$ combined with abnormal urinalysis results and numerous possible signs and symptoms of UTI, which underscores the difficulties of diagnosing true infection in this population.

Interestingly, there were no clear differences in the composition of bacteriuria between weekly asymptomatic cultures, study visits that would meet either the IDSA or NHSN CAUTI criteria, and study visits at which participants had a caregiver diagnosis of CAUTI. It is particularly striking that the organisms present at the time of all 3 caregiver-diagnosed CAUTIs had been persisting in the participants' urine specimens for several weeks prior to symptom onset and diagnosis (see participants $G$ and $J$ in Supplemental Figure 1). Further investigation of urine specimens using metagenomics and proteomics will be necessary to determine if development of signs and symptoms of infection correlates with the presence of specific organisms - including those not detected by standard culture techniques such as fungi, anaerobic bacteria, and viruses - or to changes in the host immune response. Assessment of a larger patient population will also be necessary to determine which combinations of factors provide the greatest sensitivity and specificity for CAUTI requiring therapeutic intervention versus asymptomatic colonization.

Over-testing of urine samples, both by culture and urinalysis, has been demonstrated to have a predominantly negative impact on patient outcomes, including inappropriate antimicrobial prescription and increased duration of hospitalization (66-72). For instance, detection of pyuria in preoperative urinalysis was recently demonstrated to be associated with prescription of antimicrobials even in the absence of a positive urine culture or urinary symptoms (73). Importantly, pyuria itself was not associated with any negative postoperative outcomes, but the resulting antimicrobial use increased risk of subsequent Clostridioides difficile infection without improving any other outcomes (73). This issue is further complicated in catheterized individuals, older adults, and those with neurogenic lower urinary tract dysfunction, for whom pyuria and positive urine cultures are common. It is also complicated in individuals who demonstrate minimal sensitivity for fever, dysuria, urgency, and pain, as this makes it difficult to differentiate a UTI from asymptomatic bacteriuria (27, $32,68,74-78)$. A recent study identified fever as the primary indication for obtaining a urine culture from catheterized individuals, even when other urinary symptoms were lacking and there were possible alternative explanations of fever (79). Abnormal urinalysis is another common indication for obtaining a urine culture in hospital settings (68), despite lack of other urinary symptoms and IDSA guidelines to the contrary. Considering the almost ubiquitous bacteriuria and abnormal urinalysis results observed in this study, coupled with a high prevalence of nonspecific signs and symptoms of possible infection, our data underscore the critical need for discovery of additional indicators of true infection in this patient population. 
Table 5. Urinalysis of catheter-associated bacteriuria

\begin{tabular}{|c|c|c|c|c|c|c|c|c|}
\hline & $\begin{array}{c}\text { Baseline } \\
\text { occurrence }\end{array}$ & $\begin{array}{c}\text { Point } \\
\text { prevalence }^{A}\end{array}$ & Patient weeks & $\begin{array}{c}\text { Weekly } \\
\text { prevalence }^{\mathrm{B}}\end{array}$ & New episodes & Incidence $^{c}$ & $\begin{array}{c}\text { Number of } \\
\text { 1-week } \\
\text { episodes }\end{array}$ & $\begin{array}{c}\text { Proportion } \\
\text { of } 1 \text {-week } \\
\text { episodes }^{D}\end{array}$ \\
\hline Leukocytes & 17 & 0.89 & 213 & 0.91 & 8 & 0.03 & 0 & 0 \\
\hline Protein & 10 & 0.53 & 131 & 0.56 & 30 & 0.13 & 13 & 0.43 \\
\hline Nitrite & 10 & 0.53 & 124 & 0.53 & 36 & 0.15 & 16 & 0.44 \\
\hline Blood & 8 & 0.42 & 75 & 0.32 & 14 & 0.06 & 12 & 0.86 \\
\hline $\mathrm{pH} \geq 7.25$ & 4 & 0.21 & 105 & 0.45 & 29 & 0.12 & 4 & 0.14 \\
\hline Ketone & 0 & 0.00 & 14 & 0.06 & 9 & 0.04 & 6 & 0.67 \\
\hline Urobilinogen & 0 & 0.00 & 2 & 0.01 & 2 & 0.01 & 2 & 1.00 \\
\hline Bilirubin & 0 & 0.00 & 2 & 0.01 & 2 & 0.01 & 2 & 1.00 \\
\hline Glucose & 0 & 0.00 & 0 & 0.00 & 0 & 0.00 & 0 & 0.00 \\
\hline
\end{tabular}

${ }^{A}$ Number of baseline urine urinalysis finding divided by the total number of baseline urine samples. ${ }^{B}$ Number of patient weeks urinalysis finding was detected divided by total number of patient weeks. ${ }^{C}$ Number of new instances of urinalysis finding divided by total number of patient weeks. ${ }^{\mathrm{D}} \mathrm{Number}$ of episodes lasting only 1 week divided by the number of new episodes (excluding the first and last study visits).

Frequent exposure to antimicrobials has been demonstrated to result in a high carriage rate of antimicrobial resistant organisms in nursing home residents, particularly those with indwelling devices $(8,10,11)$. While only 6 of the 19 participants received antimicrobials during the course of the study, 12 participants $(63 \%)$ were colonized by at least 1 bacterium that was resistant to a tested antimicrobial, and $26 \%$ of the 234 urine cultures contained at least 1 resistant bacterium. The persistence of antimicrobial-resistant isolates was also striking. For instance, of the 9 participants with MRSA bacteriuria, 4 exhibited MRSA for at least 8 weeks despite multiple catheter changes, and similar trends were observed for resistant $\mathrm{Gram}^{-}$bacteria.

Regarding persistent colonization, our data indicate that bacteriuria remains remarkably stable in individuals with long-term urinary catheters, even after numerous catheter changes, and antimicrobial treatment only transiently reduced colonization of some of the organisms. It is alarming that antibiotic use did not sterilize the urine for any of the study participants, with the exception of a single urine sample taken from participant $G$ while they were undergoing several weeks of i.v. antibiotic exposure. While antibiotic use often resulted in an immediate loss of 1 or 2 colonizing organisms, the net change in colonization favored an eventual increase back to the same total number of species present prior to treatment, and it often included new acquisition of a resistant bacterium. Changing the catheter upon initiation of antimicrobial treatment has been suggested to expand the duration of posttreatment culture- bacteriuria (80), but there is no clear improvement of clinical outcomes (81). Catheter insertions can also have a substantial negative impact due to the risk of creating a false passage, bladder perforation, external trauma, and hematuria, in addition to potentially inducing symptomatic CAUTI (71). Thus, our exploratory data suggest that catheter changes in long-term catheterized nursing home residents with asymptomatic bacteriuria may impose a risk of increased acquisition of bacteria, as well as new onset of signs and symptoms that are often considered to be indicators of infection without substantially reducing bacterial burden. It is important to note that not all of the reported catheter changes could have been avoided, as 13 of the 54 changes $(24 \%)$ were due to catheter obstruction or blockage and $5(9 \%)$ were due to accidental removal or dislodging of the catheter. However, the remaining $36(67 \%)$ were reported as routine care and could potentially have been avoided. While further studies are necessary, these preliminary observations provide support for catheter care practices of only changing the catheter when necessary and strictly adhering to stewardship guidelines.

The prevalence of polymicrobial bacteriuria during long-term catheterization has been widely reported for decades $(1,2,50)$. However, polymicrobial clinical urine specimens are often suspected of harboring periurethral or vaginal microbiota, particularly when they include $\mathrm{Gram}^{+}$organisms $(82,83)$. This has complicated the investigation of the clinical significance of polymicrobial bacteriuria and assessment of the contribution of these organisms to pathogenesis. It is therefore notable that prospective assessment of bacteriuria revealed that Enterococcus faecalis, Staphylococcus aureus, and coagulase- Staphylococcus are prevalent and persistent constituents of bacteriuria in nursing home residents with long-term indwelling catheters, especially as they may facilitate transient bacteremia, hematogenous seeding of other body sites, and endocarditis (84). In elderly catheterized individuals, $\sim 4 \%$ of catheter changes were demonstrated to result 
Table 6. Colonization dynamics

\begin{tabular}{|c|c|c|c|c|c|c|c|}
\hline Participant & Number of events & Isolates lost ${ }^{A}$ & Isolates regained ${ }^{\mathrm{B}}$ & New isolates ${ }^{c}$ & New ARO & $\begin{array}{l}\text { Incidence of new } \\
\text { AROE }^{\mathrm{E}}\end{array}$ & Net change ${ }^{F}$ \\
\hline \multicolumn{8}{|c|}{ Catheter changes } \\
\hline $\mathrm{A}$ & 7 & 7 & 4 & 6 & 0 & NA & +3 \\
\hline B & 5 & 1 & 0 & 0 & 1 & 0.17 & -1 \\
\hline C & 11 & 12 & 11 & 9 & 1 & 0.11 & +8 \\
\hline$D$ & 2 & 0 & 0 & 1 & 0 & NA & +1 \\
\hline $\mathrm{H}$ & 4 & 2 & 1 & 0 & 0 & 0.00 & -1 \\
\hline 1 & 2 & 0 & 0 & 1 & 0 & NA & +1 \\
\hline Average & 5.0 & 3.5 & 2.5 & 2.9 & 0.6 & 0.28 & +1.9 \\
\hline \multicolumn{8}{|c|}{ Antimicrobial treatments } \\
\hline B & 1 & 5 & 3 & 2 & 0 & 0.00 & 0 \\
\hline
\end{tabular}

Data pertain to all catheter changes and antimicrobial treatments with at least 3 weeks of subsequent follow-up from 9 study participants who completed at least 16 weeks of follow-up visits. ${ }^{A}$ Number of isolates present the week prior to the event and absent the week following the event. ${ }^{B}$ Number of isolates that were lost due to the event but reemerged in the subsequent 3 weeks. ${ }^{C}$ Number of isolates that were not present the week prior to the event but emerged in the subsequent 3 weeks. ${ }^{D}$ Number of new isolates that were antimicrobial resistant organisms (AROs). ENumber of new ARO acquisitions divided by total number of new acquisitions. FNet impact of the event on the number of isolates detected in the participants weekly urine samples. NA, not applicable.

in transient bacteremia, including by coagulase- Staphylococcus species $(1,85)$. Bacteremia due to $S$. aureus has also been observed in $\sim 7 \%$ of patients with $S$. aureus bacteriuria, particularly those of advanced age or residing in nursing homes (86). Furthermore, identification of $S$. aureus bacteriuria $\geq 48$ hours prior to bacteremia was associated with an increased risk of mortality (86). Further research is necessary to determine if persistent catheter-associated bacteriuria with $\mathrm{Gram}^{+}$organisms increases risk of bacteremia and hematogenous seeding of other body sites and if polymicrobial bacteriuria further modifies risk.

Overall, the most frequent and persistent cause of polymicrobial bacteriuria in this study was $E$. faecalis with $P$. mirabilis. The association of these organisms may have important clinical implications, as interactions between $P$. mirabilis and $E$. faecalis increase the likelihood of developing urinary stones and bacteremia in experimental models of CAUTI $(17,60)$. Ineffective antimicrobial treatment has also been reported to be more common for polymicrobial UTI and those involving E. faecalis (23). It may therefore be hypothesized that CAUTI sequelae and mortality may be more common in cocolonized study participants than those who were not cocolonized. While the present study was not sufficiently powered to address this hypothesis, it is worth noting that 3 study participants developed urinary stones, pyelonephritis, or urosepsis (rows D, G, and J of Supplemental Figure 1); all 3 were colonized by P. mirabilis, and 2 exhibited cocolonization with E. faecalis. All 3 participants were also cocolonized by Providencia stuartii, which has similarly been shown to interact with $P$. mirabilis and enhance risk of urinary stones and bacteremia $(17,87)$. However, other cocolonized participants did not exhibit infection or sequelae (such as rows A, C, and E in Supplemental Figure 1), underscoring that these polymicrobial interactions are one of many factors that contribute to the risk of developing severe disease. Further investigation of complex polymicrobial interactions in the catheterized urinary tract is likely to provide insight into potential decolonization strategies or therapeutics to reduce the risk of progressing from asymptomatic bacteriuria to CAUTI and associated sequelae.

The results of this study should be considered in light of several strengths and weaknesses. Main strengths of the study include (a) prospective longitudinal urine culturing rather than collection of a single specimen per participant; (b) prospective assessment of possible signs and symptoms of infection; (c) weekly study visits conducted by the same study personnel; (d) weekly urine culturing and antimicrobial susceptibility testing to identify and characterize all isolates prior to any laboratory adaptation and to monitor dynamics of colonization; and (e) enrollment at 2 nursing facilities. Limitations of the study include 
the limited sample size and exploratory nature of the study, lack of a control group for assessment of the impact of catheter changes and antibiotic use, low diversity of study participants, limited duration of follow-up for some study participants, inability to conduct assessments for all participants at all study visits, and use of biochemical tests to identify bacterial isolates rather than a more sensitive technology, such as matrix-assisted laser desorption ionization-TOF mass spectrometry (MALDI-TOF MS). It is also important to note that urine specimens for culture were collected at designated weekly study visits and, therefore, occurred 7 days or fewer after catheter exchanges (3.2 days on average, 3 day median) or initiation of antibiotics (2.8 days on average, less than 1 day median), but not immediately after each event. While our study is exploratory in nature and these limitations preclude more sophisticated analysis of the relationship between catheter changes, antibiotic use, presence of specific organisms, and clinical presentation, this study still represents a significant advance in our understanding of the dynamics and epidemiology of bacteriuria in nursing home residents with long-term urinary catheters. Further investigations of this nature may reveal the host and microbial factors that provide the greatest sensitivity and specificity for CAUTI requiring therapeutic intervention versus asymptomatic colonization. If so, this information could help in refining existing tools and determining which course of action should be taken for a given patient, therefore guiding appropriate antimicrobial treatment and possibly reducing acquisition of antimicrobial resistance.

\section{Methods}

Study design. A prospective observational cohort study of asymptomatic catheter-associated bacteriuria was conducted at 2 nursing homes located in Buffalo (New York, USA) between July 2019 and March 2020. Study visits occurred at enrollment and weekly thereafter for up to 7 months. Each study visit entailed chart review by trained research staff, as well as a brief assessment of possible signs and symptoms of infection and collection of a urine specimen by 1 of 3 licensed practicing nurses (LPNs) from the Visiting Nurse Association of Western New York, based in North Tonawanda (New York, USA). Participants were withdrawn from the study upon indication that they no longer wanted to participate, removal of the indwelling catheter without replacement, transfer to a nonparticipating facility, or death. All study data and records were managed using Research Electronic Data Capture (REDCap) tools $(88,89)$, hosted through the University at Buffalo Clinical and Translational Science Institute.

Inclusion criteria. Nursing home residents at either of the 2 participating facilities were eligible for inclusion if they had an indwelling urinary catheter (Foley or suprapubic) for at least 12 months, were at least 21 years of age, and were capable of assenting to participation; furthermore, informed consent was obtained from the resident or approved decision maker for inclusion. Residents receiving end-of-life care were excluded from the study.

Data collection from chart review. Information pertaining to participant demographics, age, weight, sex, comorbidities, functional status, indication for catheterization, duration of indwelling catheter use, history of UTI, and history of antimicrobial use were obtained from participant medical records by trained research staff on the baseline visit. Chart reviews were also conducted at each weekly study visit to obtain information pertaining suspected infections, hospitalizations, urine culture results, urinalysis results, and antimicrobial prescriptions. Potential signs and symptoms of CAUTI that were recorded include fever (defined as having a single temperature $>100^{\circ} \mathrm{F}$ or repeated temperatures $>99^{\circ} \mathrm{F}$ or $>2^{\circ} \mathrm{F}$ above baseline), suprapubic or costovertebral pain or tenderness, hypotension, chills or rigors, and acute mental status change (defined as a fluctuation in behavior, inattention, disorganized thinking, or an altered level of consciousness compared with baseline) $(24,25)$.

If a study participant was temporarily transferred to a hospital, medical records from the hospital stay were utilized to obtain information pertaining to suspected infections.

Assessment of possible signs and symptoms of infection. At each study visit, an LPN collected vital signs (tympanic temperature and blood pressure) and assessed costovertebral and suprapubic pain or tenderness. The LPN and a study team member also conducted a Delirium Triage Screen (DTS) (90) at the start of each visit and a Brief Confusion Assessment Method (bCAM) (90) assessment at the end of each visit to identify altered mental status (defined as fluctuating altered mental status, including altered level of consciousness, inattention, and disorganized thinking). The LPN and study team member also administered an oral questionnaire at each study visit to determine if the participant had experienced rigors or chills, nausea, lack of appetite, or fatigue since the previous visit. The sign and symptom assessment tool utilized by the study nurses is provided in Supplemental Figure 2. 
Urine collection. Urine specimens were collected from the port of the indwelling catheter by an LPN using an aseptic technique. Briefly, the catheter tubing was clamped $\sim 12$ inches below the latex rubber port, and urine was allowed to collect for approximately 30 minutes. The catheter port was then swabbed with an alcohol wipe and allowed to dry for $\sim 30$ seconds. The needle of a sterile syringe was then inserted into the port, and urine was withdrawn and transferred into a sterile specimen jar. Urine specimens were placed in an insulated cooler with ice packs and stored therein for no more than 4 hours prior to culturing.

Processing of urine specimens. Each urine specimen was utilized for isolation and identification of colonizing bacterial species, urinalysis via 10-parameter urine reagent test strip (LW Scientific), and generation of urine glycerol stock for long-term storage and reisolation, if needed. Remaining urine was also frozen at $-80^{\circ} \mathrm{C}$ for future analyses. To determine total $\mathrm{CFU} / \mathrm{mL}$ of urine, each specimen was diluted 1:100, spiral plated on HardyCHROM UTI agar (Hardy Diagnostics) using an Eddy Jet 2 spiral pater (Neutec Group Inc.), and enumerated using a ProtoCOL 3 automated colony counter (Synbiosis). For detection of Gram ${ }^{+}$ and $\mathrm{Gram}^{-}$bacteria, a $1 \mu \mathrm{L}$ calibrated inoculating loop (Laboratory Products Sales Inc.) was used for semiquantitative streak plating on 3 types of agar from Hardy Diagnostics: Columbia CNA, Bile Esculin (BEA), and MacConkey. All distinct colonies that could be differentiated by morphology, hemolysis, or color were isolated for further analysis.

$\mathrm{Gram}^{+}$bacteria from CNA and BEA plates were tested for catalase using 30\% hydrogen peroxide and for PYR activity (Hardy Diagnostics). Isolates that were $\mathrm{PYR}^{+}$and catalase ${ }^{-}$were suspected to be Enterococcus species and identified to the species level using previously described primer sets (91). Isolates that were $\mathrm{PYR}^{-}$and catalase ${ }^{+}$were suspected to be Staphylococcus species, and subjected to Sure-Vue SELECT (Fisher Healthcare) to distinguish Staphylococcus aureus from coagulase ${ }^{-}$Staphylococcus species. $\mathrm{PYR}^{-}$and catalase isolates were suspected to be Streptococcus species and were subjected to a Streptex Latex Agglutination Test (Thermo Fisher Scientific). Suspected Streptococcus isolates that did not have a positive Streptex reaction were designated "miscellaneous PYR ${ }^{-}$catalase-Gram ${ }^{+}$isolates."

Gram- bacteria from MacConkey plates were identified to the species level whenever possible using API-20E test strips (BioMérieux). Isolates identified as Pseudomonas aeruginosa via API-20E were confirmed using previously described primer sets (92). Proteus mirabilis and Proteus vulgaris isolates were confirmed by swarming motility on blood agar plates (Hardy Diagnostics).

Isolates of a given organism from consecutive urine specimens from the same participant were assumed to be the same strain if they were the same genus and species, the API-20E biotype number varied by no more than 2 digits (for $\mathrm{Gram}^{-}$isolates), and if colony morphology and antimicrobial susceptibility profiles were consistent week to week (see below). If a strain was absent in 1 urine specimen but had been present in the preceding and following specimens from that participant, reisolation was attempted from the urine glycerol stock. If the strain still could not be detected, it was assumed to be absent from that urine specimen.

Antimicrobial susceptibility testing. Antimicrobial susceptibility was assessed by zone of growth inhibition on Mueller-Hinton agar (Hardy Diagnostics). Zone diameters indicative of susceptibility were determined using the Clinical and Laboratory Standards Institute (CLSI) breakpoints listed in the M100 30th edition $(93,94)$. Enterococcus isolates were tested for vancomycin sensitivity using Etest strips (Hardy Diagnostics), and a minimum inhibitory concentration of $\leq 4 \mu \mathrm{g} / \mathrm{mL}$ was considered susceptible. $S$. aureus isolates were tested for methicillin susceptibility using cefoxitin (Hardy Diagnostics), and susceptibility was defined as a zone diameter of $\geq 18 \mathrm{~mm}$. Gram- isolates were tested for susceptibility to ciprofloxacin (Hardy Diagnostics) ( $\geq 21 \mathrm{~mm}$ zone diameter), ceftazidime (Hardy Diagnostics) ( $\geq 18$ $\mathrm{mm}$ zone diameter), ceftazidime with clavulanate (Hardy Diagnostics) ( $\geq 20 \mathrm{~mm}$ zone diameter), and imipenem (Hardy Diagnostics) ( $\geq 15 \mathrm{~mm}$ zone diameter).

Statistics. Logistic regression models and Bayesian multilevel longitudinal models were performed using statistical software R v.4.0.1 (R Core Team 2021, https://www.r-project.org/), and JAGS (https:// cran.r-project.org/web/packages/rjags/index.html). All logistic regression models were adjusted for participant-level clustering to account for multiple samples per participant. The Bayesian longitudinal models permitted rigorous handling of missing observations in the data.

For longitudinal analysis of occurrences of microbial species (and similarly for signs and symptoms), we considered 2 Bayesian multilevel models - one for analyzing the likelihood of occurrence of each individual species (and similarly individual signs or symptoms), and another for explaining the total number of microbial occurrences (and similarly, sign and symptom occurrences). In the following, we describe the 2 models used for microbial occurrences; analogous models were used for signs and symptoms. 
In the first model (model - 1), we quantified the effects of all microbial species, catheter changes, and antibiotic administration on the occurrence of each individual microbial species through a multilevel logistic regression model of the form:

$$
\begin{gathered}
\operatorname{logit} \mathrm{P}\left(y_{i t}=1\right)=\mu+\beta_{\text {cath }} \text { cath }_{i, t}+\beta_{\text {antibio }_{\text {antibio }}, t} ; \mathrm{t}=1 ; \\
\operatorname{logit} \mathrm{P}\left(y_{i t}=1\right)=\mu+\alpha(t-1)+\gamma y_{i, t-1}+\sum_{j} \beta_{j} x_{i, t-1}^{(j)}+\beta_{\text {cath }} \text { cath }_{i, t}+ \\
\beta_{\text {antibio }} \text { antibio }_{i, t} ; \mathrm{t} \geq 1 ;
\end{gathered}
$$

where, for an individual $(i)$ at time point $(t), y_{i t}$ denotes the binary indicator of a specific response microbe (e.g., MRSA); $x_{i, t-1}^{(j)}$ denotes the binary $0-1$ indicator of the $j$-th microbe other than the response microbe at individual $i$ at time $t-1$, and cath $_{i, t}$ and antibio ${ }_{i, t}$ denote the binary $0-1$ indicators of catheter changes and antibiotic administration, respectively. The parameter $\alpha$ quantifies the effect of time, the parameters $\gamma$ and $\beta_{j}$ quantify the logarithms of the OR of the occurrence of the species under consideration, due to one unit change (from 0 ["No"] to 1 ["Yes"]; while keeping other predictor variables fixed) in the binary presence status of the same species (e.g., MRSA) and j-th other species at the previous visit, and the parameters $\beta_{\text {cath }}$ and $\beta_{\text {antibio }}$ measure similar log OR due to 1 unit change (while keeping other predictor variables fixed) in the binary presence status indicators of catheter change and antibiotic administration, respectively, and for $0<p<1, \operatorname{logit} \mathrm{p}=\log \frac{p}{1-p}$. Note that, through the above logistic regression model, missingness in the response microbial species occurrence is naturally addressed within a Bayesian statistical framework. To address missingness in the predictor variables, we considered independent Bernoulli distributions

$x_{i, t}^{(j)} \sim \operatorname{Bernoulli}\left(p_{j}\right) ; \operatorname{cath}_{i, t} \sim \operatorname{Bernoulli}\left(p_{\text {cath }}\right) ; \operatorname{antibio}_{i, t} \sim \operatorname{Bernoulli}\left(p_{\text {antibio }}\right)$

in the second level of the multilevel model. Finally, in the third level of the model we specified (independent, vague) prior distributions for the model parameters. The probability parameters $p_{j}$, $p_{\text {cath }}$, and $p_{\text {antibio }}$ were assigned independent flat Uniform $(0,1)$ prior distributions. The intercept $\mu$ was assigned an independent $\operatorname{Normal}\left(0,100^{2}\right)$ prior; the regression parameters $\beta_{\text {cat }}, \beta_{\text {antibio }}, \alpha$, and $\gamma$ were assigned independent scale mixture normal priors of the form $\beta_{\text {cath }} \sim \operatorname{Normal}\left(0, \sigma_{\text {cath }}^{2}\right)$ (similarly for $\beta_{\text {antibio }}, \alpha$, and $\gamma$ ). Finally, for the regression coefficients of all 'other' microbes, induced shrinkage normal scale mixture priors of the form

$\beta_{j}=\frac{\widetilde{\beta}_{j}}{\operatorname{sd}\left(x_{i, t}^{(j)}\right)} ; \tilde{\beta}_{j} \sim \operatorname{Normal}\left(0, \sigma_{\beta}^{2}\right) ; \sigma_{\text {cath }}^{2} \sim$ inverse-Gamma $(0.001,0.001)$ were considered

In the second model (model -2 ), assuming exchangeability of the occurrence of individual microbial species at a given time point in a specific individual, we considered a multilevel Binomial regression model of the form

$$
\begin{gathered}
n_{i, t} \sim \operatorname{Binomial}\left(M, p_{i, t}\right) \\
\operatorname{logit} p_{i, t}=\mu+\beta_{\text {cath }} \text { cath }_{i, t}+\beta_{\text {antibio }_{\text {antibio }}, t} ; \mathrm{t}=1
\end{gathered}
$$

$\operatorname{logit} p_{i, t}=\mu+\alpha(t-1)+\lambda n_{i, t-1}+\beta_{\text {cath }}$ cath $_{i, t}+\beta_{\text {antibio }}$ antibio $_{i, t} ; \mathrm{t} \geq 2$ where $n_{i, t}$ is the total number (counts) of observed microbial species and $p_{i, t}$ is the corresponding probability of observing 1 generic microbial species, in subject $i$ at time $t$, and $M$ denotes the total number of microbial species considered in the study $(M=21)$. Here, $\lambda$ quantifies the log OR of the occurrence of a generic microbial species at a time $t$ in individual $i$ for a 1 unit increase in the microbial species counts in the same individual at the previous visit, and the remaining parameters have analogous interpretations as in model -1 , with a specific response microbial species being replaced by a generic species. Similar to model -1 , missingness in the response $n_{i, t}$ are addressed naturally through the model in a Bayesian framework. Missingness in the catheter change and antibiotic administration data are addressed through a similar second (Bernoulli) level: $\operatorname{cath}_{i, t} \sim \operatorname{Bernoulli}\left(p_{\text {cath }}\right)$; antibio $_{i, t} \sim \operatorname{Bernoulli}\left(p_{\text {antibio }}\right)$

In the final level, independent vague prior distributions similar to model -1 are considered for the model parameters.

We first fitted model - 1 separately on each response microbial species (and similarly on each response sign or symptom), and we then fitted model -2 collectively on all species (and similarly on all signs and symptoms) by approximating their respective posterior distributions through 10,000 Markov chain Monte Carlo (MCMC) draws, after discarding 10,000 burn-in draws, all generated using JAGS. 
In a typical Bayesian fashion, missing values in the data were augmented with the respective posterior distributions during the analysis based on the models described above, and they were subsequently integrated (marginalized) out. Finally, using the MCMC draws, the resulting (marginal) posterior distributions of the various model parameters of interest were summarized through their posterior medians and $95 \%$ credible intervals (computed through 0.025 th and 0.975 th posterior quantiles). These summaries are displayed as forest plots in Supplemental Figures 5 and 6.

Study approval. This study was approved by the University at Buffalo IRB (STUDY00002526) and complied with the provisions of the Declaration of Helsinki, Good Clinical Practice guidelines, and local laws and regulations. All participants (or approved decision makers) provided written informed consent prior to initiation of investigation, and all participants also assented to being in the study.

\section{Author contributions}

CEA conceived of, designed, obtained funding for, and oversaw the study. CEA, ALB, and MSH collected and analyzed samples, validated the methods, and curated the data. JS and SC conducted the statistical analyses, and CEA and SC interpreted the results. CEA drafted the manuscript. All authors edited and revised the manuscript and approved the final version.

\section{Acknowledgments}

We would like to thank members of the Department of Microbiology \& Immunology, the Division of Infectious Diseases, and Witebsky Center for Microbial Pathogenesis and Immunology for helpful comments and critiques. We would also like to thank the nursing home administrators and staff, as well as the Visiting Nurse Association of Western New York. This work was supported by the NIH via the National Institute of Diabetes Digestive and Kidney Diseases (R00 DK105205 and R01 DK123158 to CEA) and the National Center for Advancing Translational Sciences (UL1 TR001412 to the University at Buffalo). The sponsors were not involved in the study design, methods, subject recruitment, data collection, analysis, or preparation of the paper. The content is solely the responsibility of the authors and does not necessarily represent the official views of the funders.

Address correspondence to: Chelsie E. Armbruster, 955 Main Street, Room 5218, Buffalo, New York 14203, USA. Phone: 716.829.6059; Email: chelsiea@buffalo.edu.

1. Hooton TM, et al. Diagnosis, prevention, and treatment of catheter-associated urinary tract infection in adults: 2009 International Clinical Practice Guidelines from the Infectious Diseases Society of America. Clin Infect Dis. 2010;50(5):625-663.

2. Nicolle LE. Urinary catheter-associated infections. Infect Dis Clin North Am. 2012;26(1):13-27.

3. Dudeck MA, et al. National Healthcare Safety Network report, data summary for 2013, device-associated module. Am J Infect Control. 2015;43(3):206-221.

4. Rogers MAM, et al. Use of urinary collection devices in skilled nursing facilities in five states. J Am Geriatr Soc. 2008;56(5):854-861.

5. Tsan L, et al. Nursing home-associated infections in Department of Veterans Affairs community living centers. Am J Infect Control. 2010;38(6):461-466.

6. Kunin CM, et al. The association between the use of urinary catheters and morbidity and mortality among elderly patients in nursing homes. Am J Epidemiol. 1992;135(3):291-301.

7. Crnich CJ, Drinka P. Medical device-associated infections in the long-term care setting. Infect Dis Clin North Am. 2012;26(1):143-164.

8. Nicolle LE. Catheter associated urinary tract infections. Antimicrob Resist Infect Control. 2014;3:23.

9. Mody L, et al. A National Implementation Project to prevent catheter-associated urinary tract infection in nursing home residents. JAMA Intern Med. 2017;177(8):1154-1162.

10. Mody L, et al. A targeted infection prevention intervention in nursing home residents with indwelling devices: a randomized clinical trial. JAMA Intern Med. 2015;175(5):714-723.

11. Wang L, et al. Infection rate and colonization with antibiotic-resistant organisms in skilled nursing facility residents with indwelling devices. Eur J Clin Microbiol Infect Dis. 2012;31(8):1797-1804.

12. Garcia MM, et al. Traditional Foley drainage systems--do they drain the bladder? J Urol. 2007;177(1):203-207.

13. Willette PA, Coffield SK. Current trends in the management of difficult urinary catheterizations. West J Emerg Med. 2012;13(6):472-478.

14. Armbruster C, et al. Pathogenesis of Proteus mirabilis infection. EcoSal Plus. 2018;8(1):10.1128/ecosalplus.ESP-0009-2017.

15. Hofseth LJ, et al. Micronucleus frequencies in urothelial cells of catheterized patients with chronic bladder inflammation. Mutat Res. 1996;352(1-2):65-72.

16. Anderson RU. Response of bladder and urethral mucosa to catheterization. JAMA. 1979;242(5):451-453.

17. Armbruster CE, et al. The pathogenic potential of Proteus mirabilis is enhanced by other uropathogens during polymicrobial urinary tract infection. Infect Immun. 2017;85(2):e00808-16.

18. Nicolle LE. Catheter-related urinary tract infection: practical management in the elderly. Drugs Aging. 2014;31(1):1-10. 
19. Daniels KR, et al. Trends in catheter-associated urinary tract infections among a national cohort of hospitalized adults, 20012010. Am J Infect Control. 2014;42(1):17-22.

20. Warren JW, et al. Fever, bacteremia, and death as complications of bacteriuria in women with long-term urethral catheters. $J$ Infect Dis. 1987;155(6):1151-1158.

21. Bootsma AM, et al. Urinary incontinence and indwelling urinary catheters in acutely admitted elderly patients: relationship with mortality, institutionalization, and functional decline. J Am Med Dir Assoc. 2013;14(2):147.e7-147.12.

22. Chant C, et al. Relationship of catheter-associated urinary tract infection to mortality and length of stay in critically ill patients: a systematic review and meta-analysis of observational studies. Crit Care Med. 2011;39(5):1167-1173.

23. Esparcia A, et al. Influence of inadequate antimicrobial therapy on prognosis in elderly patients with severe urinary tract infections. Eur J Intern Med. 2014;25(6):523-527.

24. Loeb M, et al. Development of minimum criteria for the initiation of antibiotics in residents of long-term-care facilities: results of a consensus conference. Infect Control Hosp Epidemiol. 2001;22(2):120-124.

25. Stone ND, et al. Surveillance definitions of infections in long-term care facilities: revisiting the McGeer criteria. Infect Control Hosp Epidemiol. 2012;33(10):965-977.

26. Fekete T. Catheter-associated urinary tract infection in adults. https://www.uptodate.com/contents/catheter-associated-urinary-tract-infection-in-adults. Accessed September 3, 2021.

27. Nicolle LE, et al. Clinical Practice Guideline for the management of asymptomatic bacteriuria: 2019 Update by the Infectious Diseases Society of Americaa. Clin Infect Dis. 2019;68(10):1611-1615.

28. CDC. National Center for Emerging and Zoonotic INfectious Diseases (NCEZID): DHQP. https://www.cdc.gov/ncezid/whowe-are/ncezid-divisions/dhqp.html. Accessed September 3, 2021.

29. High K. Approach of infection in the older adult. http://www.uptodate.com/contents/evaluation-of-infection-in-the-older-adult?source=see_link. Accessed September 3, 2021.

30. Mouton CP, et al. Common infections in older adults. Am Fam Physician. 2001;63(2):257-268

31. Armbruster CE, et al. How often do clinically diagnosed catheter-associated urinary tract infections in nursing home residents meet standardized criteria? J Am Geriatr Soc. 2017;65(2):395-401.

32. Tambyah PA, Maki DG. Catheter-associated urinary tract infection is rarely symptomatic: a prospective study of 1,497 catheterized patients. Arch Intern Med. 2000;160(5):678-682.

33. Cope $\mathrm{M}$, et al. Inappropriate treatment of catheter-associated asymptomatic bacteriuria in a tertiary care hospital. Clin Infect Dis 2009;48(9):1182-1188.

34. Kizilbash QF, et al. Bacteremia and mortality with urinary catheter-associated bacteriuria. Infect Control Hosp Epidemiol. 2013;34(11):1153-1159.

35. Shortliffe LMD, McCue JD. Urinary tract infection at the age extremes: pediatrics and geriatrics. Am J Med. 2002;113(suppl $1 \mathrm{a)}: 55 \mathrm{~S}-66 \mathrm{~S}$.

36. Mayne S, et al. Confusion strongly associated with antibiotic prescribing due to suspected urinary tract infections in nursing homes. J Am Geriatr Soc. 2018;66(2):274-281.

37. Nicolle LE. Asymptomatic bacteriuria and bacterial interference. Microbiol Spectr. 2015;3(5).

38. Nace DA, et al. Clinical uncertainties in the approach to long term care residents with possible urinary tract infection. $J \mathrm{Am} M e d$ Dir Assoc. 2014;15(2):133-139.

39. Agata ED, et al. Challenges in assessing nursing home residents with advanced dementia for suspected urinary tract infections. J Am Geriatr Soc. 2013;61(1):62-66.

40. Juthani-Mehta M, et al. Interobserver variability in the assessment of clinical criteria for suspected urinary tract infection in nursing home residents. Infect Control Hosp Epidemiol. 2008;29(5):446-449.

41. Juthani-Mehta M, et al. Clinical features to identify urinary tract infection in nursing home residents: a cohort study. $J$ Am Geriatr Soc. 2009;57(6):963-970.

42. Dedeic-Ljubovic A, Hukic M. Catheter-related urinary tract infection in patients suffering from spinal cord injuries. Bosn J Basic Med Sci. 2009;9(1):2-9.

43. Ronald A. The etiology of urinary tract infection: traditional and emerging pathogens. Dis Mon. 2003;49(2):71-82.

44. Siegman-Igra Y, et al. Polymicrobial and monomicrobial bacteraemic urinary tract infection. J Hosp Infect. 1994;28(1):49-56.

45. Kunin CM. Blockage of urinary catheters: role of microorganisms and constituents of the urine on formation of encrustations. J Clin Epidemiol. 1989;42(9):835-842.

46. Rahav G, et al. Molecular epidemiology of catheter-associated bacteriuria in nursing home patients. J Clin Microbiol. 1994;32(4):1031-1034

47. Mobley HLT, Warren JW. Urease-positive bacteriuria and obstruction of long-term urinary catheters. J Clin Microbiol. 1987;25(11):2216-2217.

48. Breitenbucher RB. Bacterial changes in the urine samples of patients with long-term indwelling catheters. Arch Intern Med 1984;144(8):1585-1588.

49. Warren JW, et al. A prospective microbiologic study of bacteriuria in patients with chronic indwelling urethral catheters. $J$ Infect Dis. 1982;146(6):719-723.

50. Nicolle LE. Catheter-related urinary tract infection. Drugs Aging. 2005;22(8):627-639.

51. Armbruster CE, Mobley HLT. Merging mythology and morphology: the multifaceted lifestyle of Proteus mirabilis. Nat Rev Microbiol. 2012;10(11):743-754.

52. Jonsson K, et al. Urine cultures from indwelling bladder catheters in nursing home patients: a point prevalence study in a Swedish county. Scand J Urol Nephrol. 2011;45(4):265-269.

53. Fourcade C, et al. A comparison of monomicrobial versus polymicrobial Enterococcus faecalis bacteriuria in a French University Hospital. Eur J Clin Microbiol Infect Dis. 2015;34(8):1667-1673.

54. Schaeffer AJ. Catheter-associated bacteriuria. Urol Clin North Am. 1986;13(4):735-747.

55. Lawton MP, Brody EM. Assessment of older people: self-maintaining and instrumental activities of daily living. Gerontologist. 1969;9(3):179-186. 
56. Murray JL, et al. Mechanisms of synergy in polymicrobial infections. J Microbiol. 2014;52(3):188-199.

57. Nair N, et al. Impact of Staphylococcus aureus on pathogenesis in polymicrobial infections. Infect Immun. 2014;82(6):2162-2169.

58. Short FL, et al. Polybacterial human disease: the ills of social networking. Trends Microbiol. 2014;22(9):508-516.

59. Tay WH, et al. Polymicrobial-host interactions during infection. J Mol Biol. 2016;428(17):3355-3371.

60. Learman BS, et al. A Rare opportunist, Morganella morganii, decreases severity of polymicrobial catheter-associated urinary tract infection. Infect Immun. 2019;88(1):e00691-19.

61. Hajishengallis G, Lamont RJ. Dancing with the stars: how choreographed bacterial interactions dictate nososymbiocity and give rise to keystone pathogens, accessory pathogens, and pathobionts. Trends Microbiol. 2016;24(6):477-489.

62. Lin LI. A concordance correlation coefficient to evaluate reproducibility. Biometrics. 1989;45(1):255-268.

63. Loeb M, et al. Optimizing antibiotics in residents of nursing homes: protocol of a randomized trial. BMC Health Serv Res. 2002;2(1):17.

64. Loeb M, et al. Antibiotic use in Ontario facilities that provide chronic care. J Gen Intern Med. 2001;16(6):376-383.

65. Nicolle LE, et al. Infectious Diseases Society of America guidelines for the diagnosis and treatment of asymptomatic bacteriuria in adults. Clin Infect Dis. 2005;40(5):643-654.

66. Smith M-A, et al. Association of Increased Colony-Count Threshold for urinary pathogens in hospitalized patients with antimicrobial treatment. JAMA Intern Med. 2019;179(7):990-992.

67. Leis JA, et al. Reducing antimicrobial therapy for asymptomatic bacteriuria among noncatheterized inpatients: a proof-of-concept study. Clin Infect Dis. 2014;58(7):980-983.

68. Petty LA, et al. Risk factors and outcomes associated with treatment of asymptomatic bacteriuria in hospitalized patients. JAMA Intern Med. 2019;179(11):1519-1527.

69. Spivak ES, et al. Management of bacteriuria in veterans affairs hospitals. Clin Infect Dis. 2017;65(6):910-917.

70. Hartley S, et al. Overtreatment of asymptomatic bacteriuria: identifying targets for improvement. Infect Control Hosp Epidemiol. 2015;36(4):470-473.

71. Leuck A-M, et al. Complications of foley catheters--is infection the greatest risk? J Urol. 2012;187(5):1662-1666.

72. Das R, et al. Adverse outcomes in nursing home residents with increased episodes of observed bacteriuria. Infect Control Hosp Epidemiol. 2011;32(1):84-86.

73. Gupta K, et al. How testing drives treatment in asymptomatic patients: level of pyuria directly predicts probability of antimicrobial prescribing. Clin Infect Dis. 2020;71(3):614-621

74. Tambyah PA, Maki DG. The relationship between pyuria and infection in patients with indwelling urinary catheters: a prospective study of 761 patients. Arch Intern Med. 2000;160(5):673-677.

75. Orr PH, et al. Febrile urinary infection in the institutionalized elderly. Am J Med. 1996;100(1):71-77.

76. Kunin CM, et al. Morbidity and mortality associated with indwelling urinary catheters in elderly patients in a nursing homeconfounding due to the presence of associated diseases. J Am Geriatr Soc. 1987;35(11):1001-1006.

77. Mody L, Juthani-Mehta M. Urinary tract infections in older women: a clinical review. JAMA. 2014;311(8):844-854.

78. Tornic J, et al. The challenge of asymptomatic bacteriuria and symptomatic urinary tract infections in patients with neurogenic lower urinary tract dysfunction. J Urol. 2020;203(3):579-584.

79. Tedja R, et al. Catheter-associated urinary tract infections in intensive care unit patients. Infect Control Hosp Epidemiol. 2015;36(11):1330-1334.

80. Raz R, et al. Chronic indwelling catheter replacement before antimicrobial therapy for symptomatic urinary tract infection. $J$ Urol. 2000;164(4):1254-1258.

81. Babich T, et al. Replacement of urinary catheter for urinary tract infections: a prospective observational study. J Am Geriatr Soc. 2018;66(9):1779-1784.

82. Kline KA, Lewis AL. Gram-positive uropathogens, polymicrobial urinary tract infection, and the emerging microbiota of the urinary tract. Microbiol Spectr. 2016;4(2):10.1128/microbiolspec.UTI-0012-2012.

83. Hooton TM. Clinical practice. Uncomplicated urinary tract infection. NEngl J Med. 2012;366(11):1028-1037.

84. Chenoweth CE, et al. Diagnosis, management, and prevention of catheter-associated urinary tract infections. Infect Dis Clin North Am. 2014;28(1):105-119.

85. Bregenzer T, et al. Low risk of bacteremia during catheter replacement in patients with long-term urinary catheters. Arch Intern Med. 1997;157(5):521-525.

86. Stokes W, et al. Incidence and outcomes of staphylococcus aureus bacteriuria: a population-based study. Clin Infect Dis. 2018;69(6):963-969.

87. Armbruster CE, et al. Increased incidence of urolithiasis and bacteremia during Proteus mirabilis and Providencia stuartii coinfection due to synergistic induction of urease activity. J Infect Dis. 2014;209(10):1524-1532.

88. Harris PA, et al. The REDCap consortium: building an international community of software platform partners. J Biomed Inform. 2019;95:103208

89. Harris PA, et al. Research electronic data capture (REDCap)--a metadata-driven methodology and workflow process for providing translational research informatics support. J Biomed Inform. 2009;42(2):377-381.

90. Han JH, et al. Diagnosing delirium in older emergency department patients: validity and reliability of the delirium triage screen and the brief confusion assessment method. Ann Emerg Med. 2013;62(5):457-465.

91. Layton BA, et al. Enterococcus species distribution among human and animal hosts using multiplex PCR. J Appl Microbiol. 2010;109(2):539-547.

92. Spilker T, et al. PCR-based assay for differentiation of Pseudomonas aeruginosa from other Pseudomonas species recovered from cystic fibrosis patients. J Clin Microbiol. 2004;42(5):2074-2079.

93. Weinstein MP, et al. M100: Performance Standards for Antimicrobial Susceptibility Testing. https://clsi.org/media/1469/ m100s27_sample.pdf. Accessed September 3, 2021.

94. Humphries RM, et al. Understanding and addressing CLSI breakpoint revisions: a primer for clinical laboratories. J Clin Microbiol. 2019;57(6):e00203-19. 